\title{
High Resolution Mapping of QTLs for Heat Tolerance in Rice Using a 5K SNP Array
}

\author{
Shanmugavadivel PS ${ }^{1,2}$, Amitha Mithra SV 1 , Chandra Prakash', Ramkumar MK1', Ratan Tiwari ${ }^{3}$, Trilochan Mohapatra ${ }^{4}$
} and Nagendra Kumar Singh ${ }^{1 *}$ (1)

\begin{abstract}
Background: Heat stress is one of the major abiotic threats to rice production, next to drought and salinity stress. Incidence of heat stress at reproductive phase of the crop results in abnormal pollination leading to floret sterility, low seed set and poor grain quality. Identification of QTLs and causal genes for heat stress tolerance at flowering will facilitate breeding for improved heat tolerance in rice. In the present study, we used $272 \mathrm{~F}_{8}$ recombinant inbred lines derived from a cross between Nagina22, a well-known heat tolerant Aus cultivar and IR64, a heat sensitive popular Indica rice variety to map the QTLs for heat tolerance.

Results: To enable precise phenotyping for heat stress tolerance, we used a controlled phenotyping facility available at ICAR-Indian Institute of Wheat and Barley Research, Karnal, India. Based on 'days to 50\% flowering' data of the RILs, we followed staggered sowing to synchronize flowering to impose heat stress at uniform stage. Using the Illumina infinium 5K SNP array for genotyping the parents and the RILs, and stress susceptibility and stress tolerance indices (SSI and STI) of percent spikelet sterility and yield per plant (g), we identified five QTLs on chromosomes 3, 5, 9 and 12. The identified QTLs explained phenotypic variation in the range of 6.27 to 21. 29\%. Of these five QTLS, two high effect QTLS, one novel (qSTIPSS9.1) and one known (qSTIY5.1/qSS/Y5.2), were mapped in less than $400 \mathrm{Kbp}$ genomic regions, comprising of 65 and 54 genes, respectively.
\end{abstract}

Conclusions: The present study identified two major QTLs for heat tolerance in rice in narrow physical intervals, which can be employed for crop improvement by marker assisted selection (MAS) after development of suitable scorable markers for breeding of high yielding heat tolerant rice varieties. This is the first report of a major QTL for heat tolerance on chromosome 9 of rice. Further, a known QTL for heat tolerance on chromosome 5 was narrowed down from $23 \mathrm{Mb}$ to $331 \mathrm{Kbp}$ in this study.

Keywords: Rice, Heat tolerance, Nagina22, QTL mapping, SNP

\section{Background}

Rice is a major staple food crop for nearly half of the world population. The global population is projected to grow from seven to nine billion by 2050 and to reach ten billion before 2100 (United Nations 2011). To ensure food security to the added population, rice production has to increase by 0.6 to $0.9 \%$ annually until 2050 (Carriger and Vallee 2007). However, rise in global average temperature to the tune of $0.5{ }^{\circ} \mathrm{C}$ in the twentieth century and future projections in the range of $1.4-5.8^{\circ} \mathrm{C}$

\footnotetext{
* Correspondence: nksingh4@gmail.com

${ }^{1}$ ICAR-National Research Centre on Plant Biotechnology, New Delhi, India Full list of author information is available at the end of the article
}

by the end of this century (IPCC 2007), will be detrimental to crop yield (Lobell et al. 2011). Declining farmland resources coupled with global warming have forced rice cultivation to marginal environments and beyond the normal rice season. This in turn has exposed the rice crop to higher day temperature $\left(>33{ }^{\circ} \mathrm{C}\right)$ adversely impacting seed set (Nakagawa et al. 2002; Prasad et al. 2006; Jagadish et al. 2010a).

Heat stress alters the initiation and duration of developmental phases, especially the duration from floral/ panicle initiation to anthesis/panicle exertion in plants (Sato et al. 1973). Heat stress during flowering and anthesis can lead to failure in fertilization due to pollen or ovule sterility (Matsui et al. 1997). Early reproductive processes viz., micro- and megasporogenesis, pollen and 
stigma viability, anthesis, pollination, pollen tube growth, fertilization, and early embryo development are all highly susceptible to heat stress (Giorno et al. 2013). Flowering stage is crucial for crop productivity as heat stress during this phase causes reduced pollen fertility and low seed set in rice (Jagadish et al. 2010a). Anthesis processes, including anther dehiscence, pollination, and pollen germination are most sensitive to high temperature stress in rice. The main cause of spikelet sterility induced by high temperature is anther indehiscence (Matsui et al. 1999). High temperature inhibits swelling of pollen grains, which is a driving force for anther dehiscence in rice. Successful anther dehiscence depends on several parameters, including rupturing of septa, expansion of locule walls, pollen swelling, and rupturing of stomium (Liu et al. 2006).

Enhanced heat tolerance in rice is required at flowering stage to avoid spikelet sterility. Since 1985, germplasm screening for high temperature stress tolerance has been carried out by different research groups worldwide (Sarwar and Avesi 1985; Matsui and Omasa 2002; Jagadish et al. 2007, 2008). Heat tolerance at flowering stage in rice is attributed to multiple genes with cumulative effects on trait expression, otherwise called as quantitative trait loci (QTL; Cao et al. 2003; Xiao et al. 2011a; Ye et al. 2012). The discovery of genes/QTLs for enhanced tolerance to high temperature stress has practical implications in agriculture. Mapping of QTLs for heat tolerance in rice was first reported by Cao et al. (2003) based on percent spikelet fertility using doubled haploid population derived from IR64/Azucena cross. Thereafter many research groups have mapped QTLs for heat stress tolerance using $F_{2}$, back cross inbred lines (BIL) and recombinant inbred lines (RIL) populations, evaluated at the time of heading in controlled environment conditions (Chang-Lan et al. 2005; Chen et al. 2008; Zhang et al. 2008, 2009; Jagadish et al. 2010b; Xiao et al. 2011a; Ye et al. 2012, 2015; Cheng et al. 2012; and Poli et al. 2013). Some of these studies created high temperature condition for phenotyping by late planting in open field (Xiao et al. 2011b; Tazib et al. 2015; and Zhao et al. 2016). Almost all of these studies employed RFLP or SSR markers, except Ye et al. (2012, 2015), who used 300 SNP markers for the QTL mapping. IR64 has been used as one of the parental lines in generating mapping populations for mapping heat stress tolerance QTLs in some studies (Cao et al. 2003; Ye et al. 2012, 2015), while Nagina22 and its derived mutant lines have been used as parents in generating mapping population by other researchers (Buu et al. 2014; Poli et al. 2013). There is a report on using IR64/Nagina22 derived $F_{2}$ population for mapping heat tolerance QTLs (Ye et al. 2012).
Mapping of QTLs for heat stress tolerance using stress tolerance indices, which compare the performance of genotypes under control and stress condition, have not been reported earlier, but it has been utilized for mapping salt stress tolerance (Fernandez 1992; Pandit et al. 2010; Tiwari et al. 2016). The relative performance of genotypes under stress and control conditions can be used as an indicator to identify and map QTLs, which can be further used in breeding crop varieties for stress tolerance, rather than mapping QTLs based on phenotypic performance in stress environment alone (Raman et al. 2012). This has practical relevance since genotypes with low yield potential under control condition quite often show higher tolerance to stress than high yielding genotypes. Genomic regions governing salinity stress tolerance was successfully mapped in rice using stress indices (Pandit et al. 2010, Kumar et al. 2015; Tiwari et al. 2016). The present study focused on identification of QTLs for heat stress tolerance at flowering stage in a RIL mapping population derived from Nagina22/IR64 cross using controlled phenotyping facility for imposing heat stress, using stress tolerance indices for normalization of intrinsic differences in yield potential and high density SNP mapping. High density linkage map is expected to result in finding QTLs flanked by closely linked markers that can be readily used in breeding programmes for marker assisted selection.

\section{Methods \\ Plant Materials}

We used $272 \quad F_{7: 8}$ RILs developed through single seed descent method from a cross between Nagina22 (N22), a heat tolerant cultivar (Mohapatra et al. 2014; Prakash et al. 2016) and IR64, a heat susceptible cultivar (Jagadish et al. 2010). To achieve synchronized flowering, the RILs were first phenotyped for days to $50 \%$ flowering and grouped into three categories as early, medium and late flowering types and then were sown in a staggered manner for synchronization of their flowering time. This exercise enabled us to impose heat treatment at a uniform stage in the population that in turn minimized the interference of phenological differences in analysis.

\section{Heat Stress Treatment}

The RILs along with the two parental lines were direct sown in the controlled temperature phenotyping facility at ICAR-Indian Institute of Wheat and Barley Research, Karnal, India in an augmented design for exposing them to high temperature at flowering stage. A plant-to-plant distance of $15 \mathrm{~cm}$ and row-to-row distance of $20 \mathrm{~cm}$ was maintained. The structure was kept open from sowing to till the flowering stage, where the experimental RILs were grown in a condition similar to that prevailing outside the green house (Fig. 1). Heat stress was imposed 
on plants during flowering time by closing the shutter. The temperature inside the structure was programmed to be $5{ }^{\circ} \mathrm{C}$ higher than the air temperature outside the structure (Additional file 1: Figure. S1). Relative humidity of $70 \%$ was maintained inside the facility. Heat stress was imposed continuously for 10 days including night time. After the treatment, the structure was kept open until harvest. The same RIL population was also raised outside the green house to phenotype their performance under control or ambient conditions.

\section{Phenotyping of the $\mathrm{F}_{8}$ RILs for Heat Stress Tolerance}

Five individual plants from each RIL were sampled and harvested separately. Main panicle from each plant was used for analysing spikelet fertility by counting the number of filled and empty spikelets. The remaining panicles were collected separately from each plant and utilised for calculating yield potential of RILs under heat stress. In the same way, five plants from each RIL were harvested from the control experiment for evaluating the performance of genotypes under ambient conditions, used for computing their stress response index. The response of genotypes to heat stress was expressed as stress susceptibility index (SSI) given by Fisher and Mauer (1978) and stress tolerant index (STI) given by Fernandez (1992). SSI assesses the reduction in yield caused by stress as compared to favourable environment. The heat tolerant genotypes would have lower SSI value, which indicates lower difference in their yield across control and stress while it would be vice-versa for the susceptible genotypes. Thus, SSI helps to identify more tolerant lines. STI, on the



Fig. 1 Phenotyping of $F_{8}$ RIL mapping population under non-stress and heat stress conditions at ICAR-IIWBR. (a) At vegetative stage under non-stress condition (b). At reproductive stage under non-stress (c). At vegetative stage under non-stress condition inside controlled environment structure with roof top open (d). With reproductive stage heat stress for 10 days in controlled environment structure with roof top covered other hand, helps to identify genotypes that produce higher yield in control as well as stress conditions, which is more desirable for practical reasons. The tolerant lines have higher STI value.

\section{Genotyping of RILs}

Genomic DNA of all RILs, and the two parents was isolated from pooled young green leaves from plants grown in a row in the field by CTAB method (Doyle and Doyle 1990). DNA was quantified using NanoDrop 8000 spectrophotometer (Thermo Scientific, USA) and concentration of DNA was adjusted to a minimum of $50 \mathrm{ng} / \mu \mathrm{l}$ and approximately $200 \mathrm{ng}$ of DNA from each genotypes were used for hybridization in Illumina Infinium ${ }^{\circ}$ II genotyping assay. A customized array with 5246 SNPs in abiotic stress responsive genes of rice employing Illumina Infinium ${ }^{\bullet}$ II design probes and dual color channel assays (Infinium HD Assay Ultra, Illumina), was used for genotyping, following the manufacturer's protocol (Kumar et al. 2015).

\section{SNP Genotype Calling}

SNP genotyping data obtained from the array were analysed using Genome Studio V2010.1 (Illumina Inc.). SNPs were called using genotyping module integrated in the software where individual SNP is viewed as GenoPlots. Data quality was confirmed with internal controls and QC functions such as GenTrain and GenCall scores. After calling the data automatically, the SNPs were rescored and checked for their presence in a canonical cluster to get a GenTrain score > 0.7. The samples with call rates of $<0.89$ and the SNPs with norm $R$ values $<0.2$ were removed from further analysis. The genotype calls from parental lines Nagina22 and IR64 were converted into AA and BB, respectively and similarly SNP calls of segregating genotypes were transformed in concordance with either parental type and used for the construction of framework linkage map.

\section{QTL Mapping and Epistatic Interaction Network}

QTL mapping was carried out using QTL IciMapping software v4.0 (Meng et al. 2015). Segregation pattern of each SNP in the RIL population was analysed using chi-square test with statistical significance at $P$ value of 0.01 . The redundant markers with identical scores were removed since they cannot provide any additional information. Markers with correlation coefficient of 1 were deleted by choosing missing proportion option. The genetic distance (cM) between SNP markers was converted to physical distance $(\mathrm{kb})$ with $1 \mathrm{cM}$ equal to $260 \mathrm{kbp}$ (Chen et al. 2002; Tiwari et al. 2016). QTLs for heat stress tolerance were mapped using BIP functionality available in the QTL IciMapping software. Inclusive composite interval mapping of additive and 
dominant QTL (ICIM-ADD) mapping method was chosen along with the following parameters viz., window size of $1 \mathrm{cM}, 500$ permutations and type I error at 0.05 to call for QTL. The LOD threshold was set at 3.5 to accept the call as a significant QTL. The epistatic interaction network was determined for all the four traits studied using MQM algorithm in R/ QTL (Browman et al. 2003).

\section{In-silico Identification of Non-synonymous SNPs in Genes Present in the Mapped QTL Intervals}

Non-synonymous SNPs between N22 and IR64 were identified using Rice SNP-Seek Database (Mansueto et al. 2017) with Nipponbare as reference genome and N22 and IR64 as query genomes. The gene locus id was given as input to retrieve the non-synonymous SNPs between N22 and IR64 for further analysis.

\section{Results}

Phenotypic Variation and Correlation Among Traits in the RIL Population

The parents and RILs were analysed for their phenotypic performance under ambient and heat stress environments. Heat sensitive parent IR64 showed very high spikelet sterility (95.94\%) under heat stress as compared to the tolerant parent N22 (67.45\%; Table 1$)$. The yield reduction under heat stress was to the tune of $66 \%$ in N22 while it was $86 \%$ in IR64. Though the RIL population mean for percent spikelet sterility and yield was skewed towards IR64, it showed transgressive segregation and had high coefficient of variation $(\mathrm{CV})$, more than 20\% under both control and stress conditions (Table 1). Since SSI and STI are better indicators of plant performance under stress, we used these indices for mapping QTLs for heat tolerance. The RIL population exhibited transgressive segregation for all the four parameters analysed, namely SSI and STI of both percent spikelet sterility and yield per plant. The STI for percent spikelet sterility ranged from 0.38 to 14 while

Table 1 Performance of the parents and their recombinant inbred lines under control and heat stress

\begin{tabular}{llllll}
\hline & \multicolumn{2}{l}{ Percent spikelet sterility } & & \multicolumn{2}{l}{ Yield per plant $(\mathrm{g})$} \\
\cline { 2 - 3 } & Control & Heat stress & & Control & Heat stress \\
\hline Nagina22 & 6.38 & 67.45 & & 6.41 & 2.18 \\
IR64 & 14.53 & 95.94 & & 9.48 & 1.33 \\
RILs & $2.17-78.01$ & $15.40-100.00$ & & $1.89-24.12$ & $0.064-12.65$ \\
Mean & 16.56 & 81.8 & & 9.47 & 2.65 \\
Range & 75.84 & 84.60 & & 22.23 & 12.59 \\
SD & 12.85 & 16.4 & & 3.19 & 2 \\
CV $^{\text {b }}$ & 77.60 & 20.05 & & 33.7 & 75.5 \\
\hline
\end{tabular}

${ }^{\mathrm{a} S t a n d a r d}$ Deviation

${ }^{\mathrm{b}}$ Coefficient of variation
SSI for percent spikelet sterility ranged from -0.11 to 5.92. Similarly, the STI for yield ranged from 0.005 to 1.34 while SSI for yield ranged from 0.0078 to 1.37 (Table 2). High CV was observed for all the traits in a range of 0.32 (SSI for yield per plant) to 0.85 (STI of yield per plant), suggesting that all the four traits were suitable for QTL mapping. As expected, significant negative correlation was observed between STI and SSI for percent spikelet sterility and, STI for percent spikelet sterility and STI for yield (Fig. 2). The only positive correlation was between SSI for percent spikelet sterility and SSI for yield per plant, which is expected.

\section{SNP Marker Segregation and Framework Linkage Map}

Out of the 5246 SNPs in the stress responsive genes genotyped using Illumina Infinium chip, 1512 were polymorphic between N22 and IR64 and segregated in the RIL population (28.82\% polymorphism; Fig. 3$)$. The highest number of polymorphic markers was present on chromosome 1 (203 SNPs) while the lowest was on chromosome 9 (66). In terms of proportion of polymorphic markers, chromosome 5 had the highest (0.35) proportion while chromosome 11 had the least $(0.23)$. Thirty eight percent of polymorphic markers did not segregate as per the expected Mendelian segregation of 1:1 at cut-off probability of 0.01 and hence they were removed from further analysis. Another 117 markers that were redundant and played no role in improving the resolution of genetic map were also removed from further analysis. The highest number of redundant markers was found on chromosome $5(24.76 \%)$ while the lowest number was on chromosome 8 (3.92\%; Table 3). After removing the redundant markers, Chromosome 1 had the maximum number of markers (127) while chromosomes 4 and 7 had the lowest number of 31 markers. Finally, 824 markers were included in the framework linkage map used for QTL mapping.

\section{QTLs for Heat Tolerance Traits}

Using inclusive composite interval mapping (ICIM) approach, a total of five QTLs for stress tolerance and stress susceptibility index for yield and percent spikelet sterility were identified (Fig. 4). One QTL each for SSI and STI of percent spikelet sterility and two and one QTLs for SSI and STI for yield, respectively were mapped on four different chromosomes, namely chromosomes 3, 5, 9, and 12 with phenotypic variation explained ranging from 6.37 to $21.29 \%$ (Table 4). qSSIY5.2 was the major QTL identified in this study for yield explaining $21.29 \%$ of the phenotypic variation while qSTIPSS9.1 was the major QTL for percent spikelet sterility which explained $16.05 \%$ phenotypic variation. Except for qSTIY5.1, the heat susceptible parent IR64, contributed the heat sensitivity allele for both the 
Table 2 Phenotypic variation for heat stress tolerance indices in Nagina22/IR 64 RIL mapping population

\begin{tabular}{|c|c|c|c|c|c|c|c|c|c|}
\hline \multirow{2}{*}{$\begin{array}{l}\text { S. } \\
\text { No. }\end{array}$} & \multirow[t]{2}{*}{ Traits } & \multicolumn{6}{|l|}{ RILS } & \multirow[t]{2}{*}{ N22 } & \multirow[t]{2}{*}{ IR64 } \\
\hline & & Minimum & Maximum & Range & Mean & $S D^{a}$ & $C V^{b}$ & & \\
\hline 1 & SSI for \% spikelet sterility & -0.10919 & 5.9246 & 6.03379 & 1.6576 & 1.2916 & 0.77919 & 2.422 & 1.418 \\
\hline 2 & STI for \% spikeletsterility & 0.38193 & 13.9979 & 13.6159 & 4.5501 & 3.1156 & 0.68473 & 1.576 & 5.106 \\
\hline 3 & STI for yield per plant & 0.00469 & 1.33855 & 1.33386 & 0.2805 & 0.2395 & 0.85383 & 0.3314 & 0.066 \\
\hline 4 & SSI for yield per plant & 0.00782 & 1.37249 & 1.36467 & 0.9764 & 0.3084 & 0.31585 & 0.9142 & 1.1918 \\
\hline
\end{tabular}

\section{${ }^{\text {a }}$ Standard Deviation}

${ }^{\mathrm{b}}$ Coefficient of variation

spikelet sterility and yield QTLs. qSTIY5.1 and qSSIY5.1 are one and the same as they are present in the same genomic region. As expected, the tolerance allele of qSSIY5.1 locus was contributed by N22 while the sensitivity allele was from IR64. Further, the additive effect of the trait enhancing allele from $\mathrm{N} 22$ was twice as that of IR64 (Table 4). Analysis of physical positions of the identified QTLs revealed that qSTIY5.1/qSSIY5.1 was in a

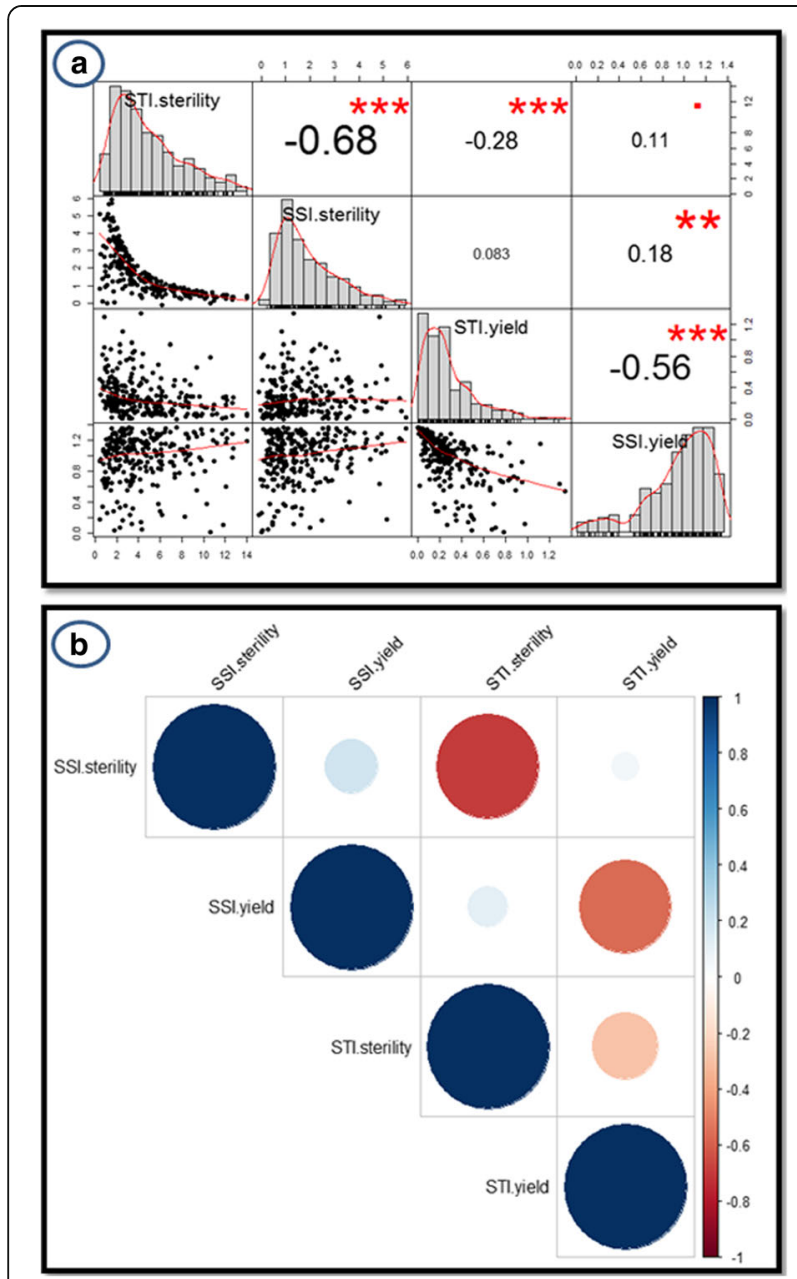

Fig. 2 Phenotype distributions and Correlation of stress indices. (a) Trait distribution and linear correlation values (b). Correlogram of stress indices small interval of $331 \mathrm{kbp}$ on chromosome 5. The other major QTL identified, qSTIPSS9.1, was also located in a small interval of $394 \mathrm{kbp}$ on chromosome 9. The largest interval of $1067.5 \mathrm{kbp}$ was for the QTL qSSIY3.1, which explained $6.45 \%$ of phenotypic variations of SSI for yield (Table 4). qSSIPSS12.1, a minor QTL for percent spikelet sterility was mapped between markers SNP14876 and SNP14892, explaining $6.37 \%$ of the phenotypic variation. As expected, the sterility enhancing allele for this QTL was from susceptible parent IR64. Thus, for all the four QTLs identified in this study, the heat tolerance allele was from the heat tolerant variety N22.

\section{Candidate Genes for Heat Tolerance Located in the QTL Intervals}

The genes located in the four genomic regions for the identified QTLs were extracted (Additional file 2: Table S1). Probable candidate genes for heat tolerance located in the major QTL interval qSTIPSS9.1 for spikelet fertility were $P T C 1$, glycosyltransferase, microtubule associated protein, annexin and HSFs. Similarly, candidate genes for heat tolerance index in the narrowed down QTL interval qSTIY5.1/qSSIY5.2 included, trehalose synthase, trehalose-6-phosphate synthase, auxin response factor and calcineurin B-like protein-interacting protein kinase (CIPKs). In the QTL interval qSSIPSS12.1, 134 genes were identified including lipases, laccase, isoflavonereductase, cyclopropane-fatty-acyl-phospholipid synthase, OGR1, wall associated receptor kinase and pentatricopeptide protein coding genes. The qSSIY3.1 QTL harboured 142 genes including, TFs, signalling genes and floral organ developmental genes, tesmin/TSO1, Crinkly4 receptor-like kinase, LIM domain containing protein gene and pectin methylesterase inhibitor coding gene. The majority of these genes are reported to be involved in pollen grain development, pollen tube growth and fertilization in rice.

\section{Digenic Interactions of QTLs for Heat Tolerance}

Four significant digenic interactions involving seven SNPs were identified for three traits (Fig. 5) namely, STI for sterility and SSI and STI for yield on chromosomes 2, 3, 4, 5, 9 and 12 (Table 5). Interestingly, STI for 


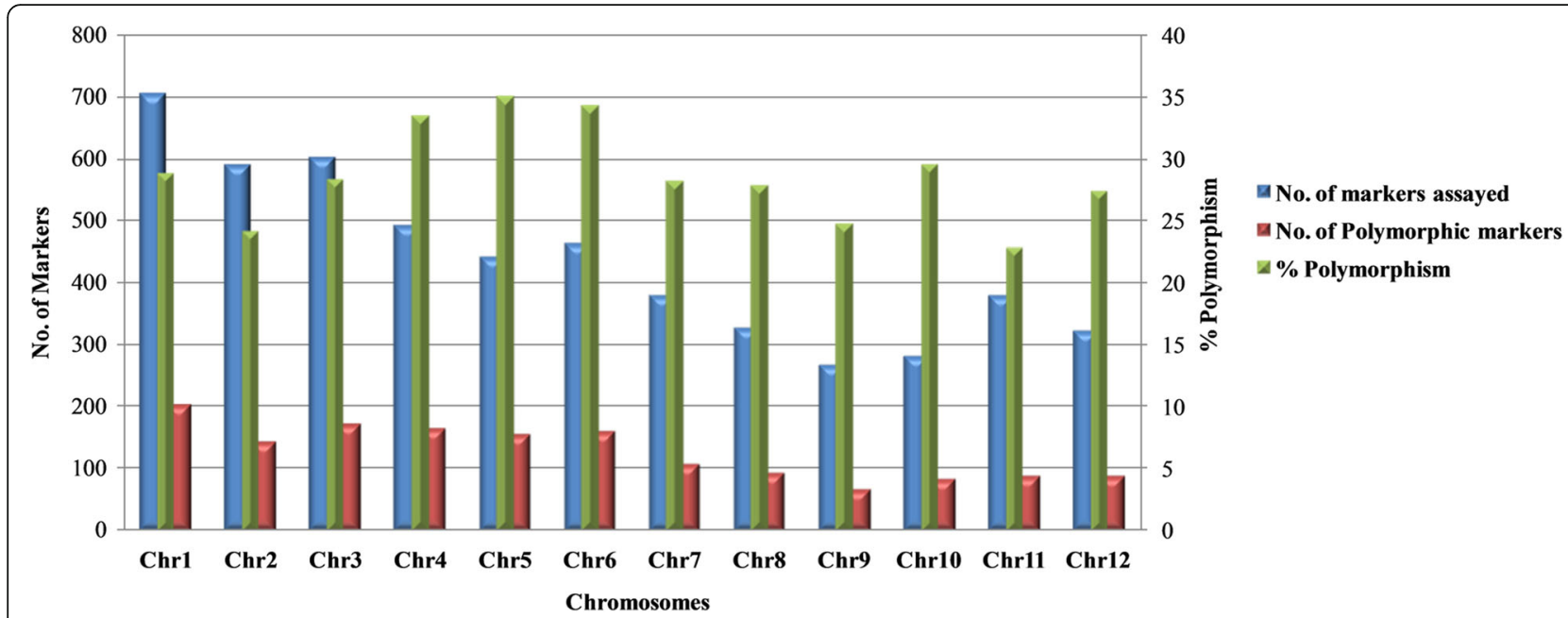

Fig. 3 Chromosome-wise polymorphism survey using 5K SNP array and polymorphic SNPs in Nagina22 x IR64 mapping population

yield had two digenic interactions involving a common SNP (7118) from a locus encoding dehydrogenase (LOC_Os04g52280). One of these interactions involved an SNP (8401) from the major and common QTL identified in the study, qSTIY5.1/qSSIY5.2. SNP8401 is present in a gene encoding WD40 domain, G-beta repeat domain containing protein (LOC_Os05g44320). All other SNPs showing epistasis were in uncharacterized expressed protein coding genes (Table 5).

\section{Non-synonymous SNPs in the mapped QTL intervals}

The maximum number of SNPs (71 SNPs in 26 genes) were observed in qSSIPSS12.1 genomic interval while the lowest number of SNPs (21 SNPs in 14 genes) were in $q$ STIY5.1 /qSSIY5.1 region (Table 6). Ty3-gypsy subclass retrotransposon protein encoding gene (LOC_Os12g17290) had the highest number of SNPs (8). Approximately, $77 \%$ of the observed substitutions were base transitions. SNPs were present in expressed genes, transcription factor coding genes, transposon related genes and protein and enzyme coding genes. qSTIPSS9.1 genomic region had 28 SNPs including 23 transitions and 5 transversions in 16 different genes. qSSIPSS12.1 interval had 71 SNPs in 26 genes including laccase precursor protein coding gene, expressed genes, Cyclopropane-fatty-acyl-phospholipid synthase coding genes and transposon related genes (Additional file 2: Table S2).

\section{Discussion}

QTLs for heat tolerance have been mapped on different chromosomes of rice by different research groups during the last decade (Cao et al. 2003; Chen et al. 2008; Zhang et al. 2008, 2009; Jagadish et al. 2010; Xiao et al. 2011; Ye et al. 2012, 2015) (Additional file 2: Table S3). In the current study, using a reasonably large RIL population, high density SNP map and phenotyping under controlled facility, we identified four heat tolerant QTLs in rice, of which three were novel namely qSTIPSS9.1, qSSIPSS12.1 and qSSIY3.1. Among these, qSTIPSS9.1 was the major QTL for percent spikelet sterility. Further, we also identified a known major effect QTL, qSSIY5.1/ qSTIY5.1 for both the indices of yield. Zhang et al. (2008) have reported this QTL in a RIL mapping population derived from a cross Zhongyouzao8 $\mathrm{x}$ Toyonishiki between SSR markers, RM405 and RM274 flanking a $23 \mathrm{Mb}$ interval. In their study, this QTL explained 10.7\% phenotypic variation for spikelet fertility under heat stress while it was for SSI/STI for yield in our study. Further, the QTL interval was narrowed down to a 331

Table 3 Selection of polymorphic markers for QTL mapping

\begin{tabular}{|c|c|c|c|c|c|c|c|c|c|c|c|c|c|}
\hline & Chr1 & Chr2 & Chr3 & Chr4 & Chr5 & Chr6 & Chr7 & Chr8 & Chr9 & Chr10 & Chr11 & Chr12 & Total \\
\hline $\begin{array}{l}\text { No. of Markers after Chi square test at } 0.01 \\
\text { significant level }\end{array}$ & 152 & 120 & 94 & 33 & 105 & 108 & 34 & 51 & 51 & 63 & 62 & 68 & 941 \\
\hline \% Segregation distortion & 25.12 & 15.49 & 44.7 & 79.87 & 31.81 & 31.64 & 68.22 & 43.95 & 22.72 & 24.09 & 27.9 & 22.72 & 37.76 \\
\hline $\begin{array}{l}\text { No. of markers per chromosome after } \\
\text { removing redundant markers }\end{array}$ & 127 & 107 & 83 & 31 & 79 & 98 & 31 & 49 & 43 & 58 & 58 & 60 & 824 \\
\hline$\%$ Redundant markers & 16.44 & 10.83 & 11.7 & 6.06 & 24.76 & 9.26 & 8.82 & 3.92 & 15.68 & 7.93 & 6.45 & 11.76 & 12.43 \\
\hline No. of markers used for QTL analysis & 127 & 107 & 83 & 31 & 79 & 98 & 31 & 49 & 43 & 58 & 58 & 60 & 824 \\
\hline
\end{tabular}




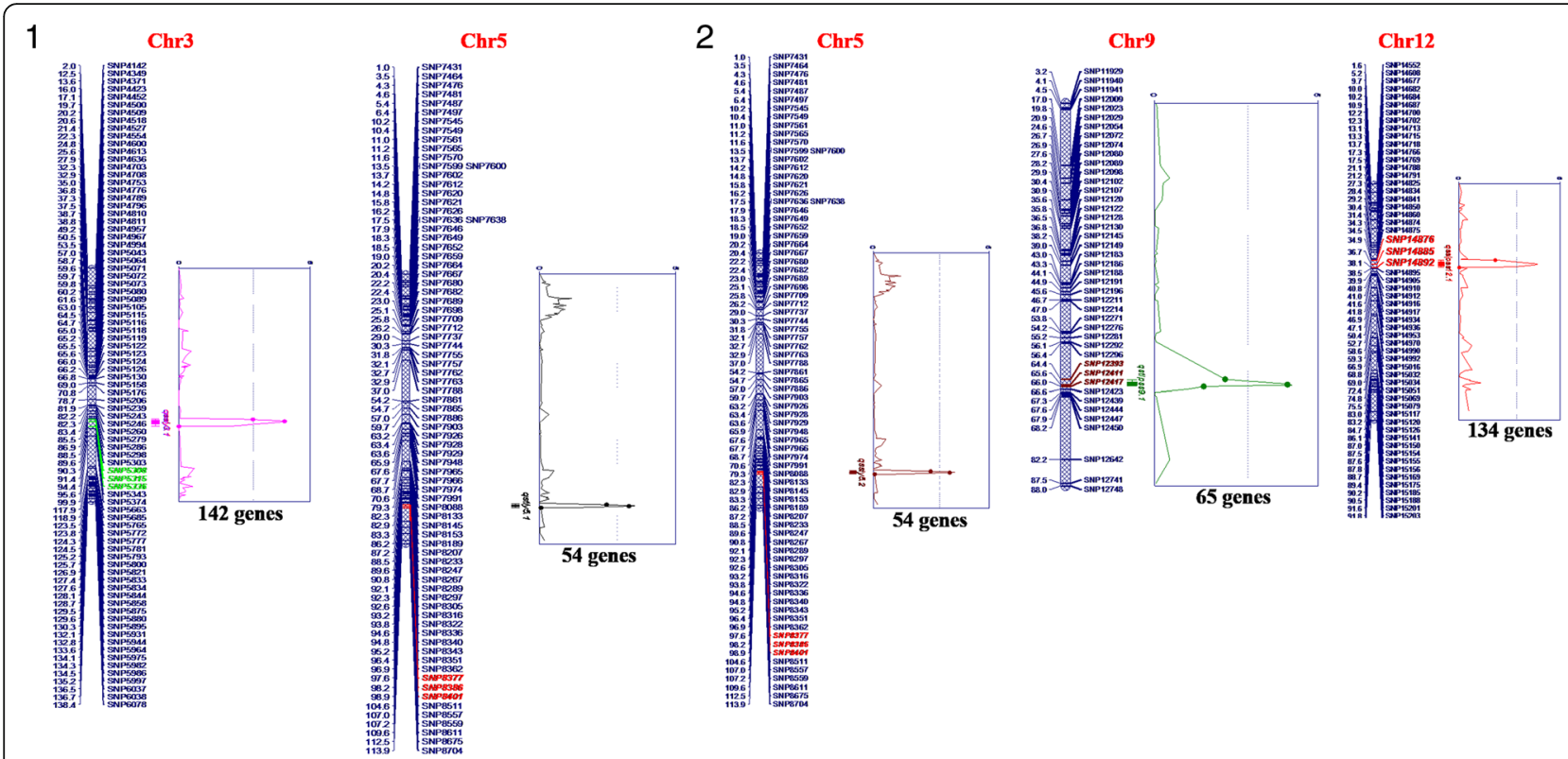

Fig.4 QTLs identified for heat tolerance in rice in the mapping population derived from Nagina22 and IR64

kbp region comprising of 54 genes in our study. This was because earlier studies have used either SSR markers (maximum 264) or less than 300 SNP markers for mapping QTLs for heat tolerance whereas we have used more than 800 SNPs and 272 RILs to achieve a much higher resolution (Buu et al. 2014; Ye et al. 2012, 2015). Our ability to identify QTLs in such narrow intervals could be attributed to the use of $5 \mathrm{~K} \mathrm{SNP}$ array comprising of SNPs from abiotic stress responsive genes (Kumar et al. 2015). Some important candidate genes located in the high effect and minor QTLs identified in the present study are discussed below for their probable role in enhanced spikelet fertility and yield under heat stress.

There were 65 genes in the QTL region, qSTIPSS9.1, including transporters, transcription factors such as $H S F$ $(O s H s f B 4 c)$, PHD-finger domain containing TF (PTC1), $b H L H$, and $C 2 H 2$ zinc finger, transcriptional regulators, glycosyltransferase microtubule associated protein, and annexin (Additional file 2: Table S1). Tapetum, the innermost cell layer of the anther wall, plays a crucial role in anther development, microspore/pollen formation, and pollen wall formation. During late pollen development, tapetal degeneration triggered by an apoptosis-like process is essential for viable pollen formation (Li et al. 2006). PERSISTENT TAPETAL CELL1 (PTC1) present in the $q$ STIPSS9.1 QTL region encodes a PHD-finger protein that controls programmed tapetal development and degradation to ensure functional pollen formation in rice ( $\mathrm{Li}$ et al. 2011a, b). PTC1 is expressed specifically in tapetal cells and microspores during anther development in stages 8 and 9 and initiates a typical apoptosis-like cell death, thereby ensuring proper pollen grain development (Li et al. 2011). Loss of function of PTC1 displayed uncontrolled tapetal cell proliferation and swelling, delayed DNA fragmentation, and pollen wall development, causing complete male sterility (Li et al. 2011). Timely initiation of tapetal programmed cell death is essential for the regulated release of wall materials from the tapetum to the developing microspore including carbohydrate, lipid molecules, and other nutrients. This gene might be responsible for maintaining higher fertility in N22 under heat stress by timely initiation of PCD in N22 tapetal cell to ensure more fertile pollen grains than in the susceptible parent IR64. This gene otherwise named as a tms $9-1 / O s M S 1$ is

Table 4 QTLs for heat stress indices identified in RIL mapping population derived from N22 and IR64

\begin{tabular}{|c|c|c|c|c|c|c|c|c|c|}
\hline Trait Name & QTL name & Chr. & Left Marker & Right Marker & Physical position (Mb) & Interval (Kb) & LOD & PVE (\%) & $\overline{\text { Add }}$ \\
\hline STI for \% spikelet sterility & QSTIPSS9.1 & 9 & SNP12393 & SNP12417 & $16.75-17.14$ & 393.828 & 4.21 & 16.05 & -1.25 \\
\hline SSI_for \% spikelet sterility & qSSIPSS12.1 & 12 & SNP14876 & SNP14892 & $9.06-9.90$ & 840.288 & 3.88 & 6.37 & -0.33 \\
\hline STI for yield per plant & qST/Y5.1 & 5 & SNP8377 & SNP8401 & $25.45-25.79$ & 331.586 & 4.55 & 9.01 & 0.07 \\
\hline SSI for yield per plant & qSSIY3.1 & 3 & SNP5308 & SNP5336 & $23.52-24.59$ & 1067.507 & 4.04 & 6.45 & -0.08 \\
\hline SSI for yield per plant & qSSIY5.1 & 5 & SNP8377 & SNP8401 & $25.45-25.79$ & 331.586 & 3.51 & 21.29 & -0.14 \\
\hline
\end{tabular}




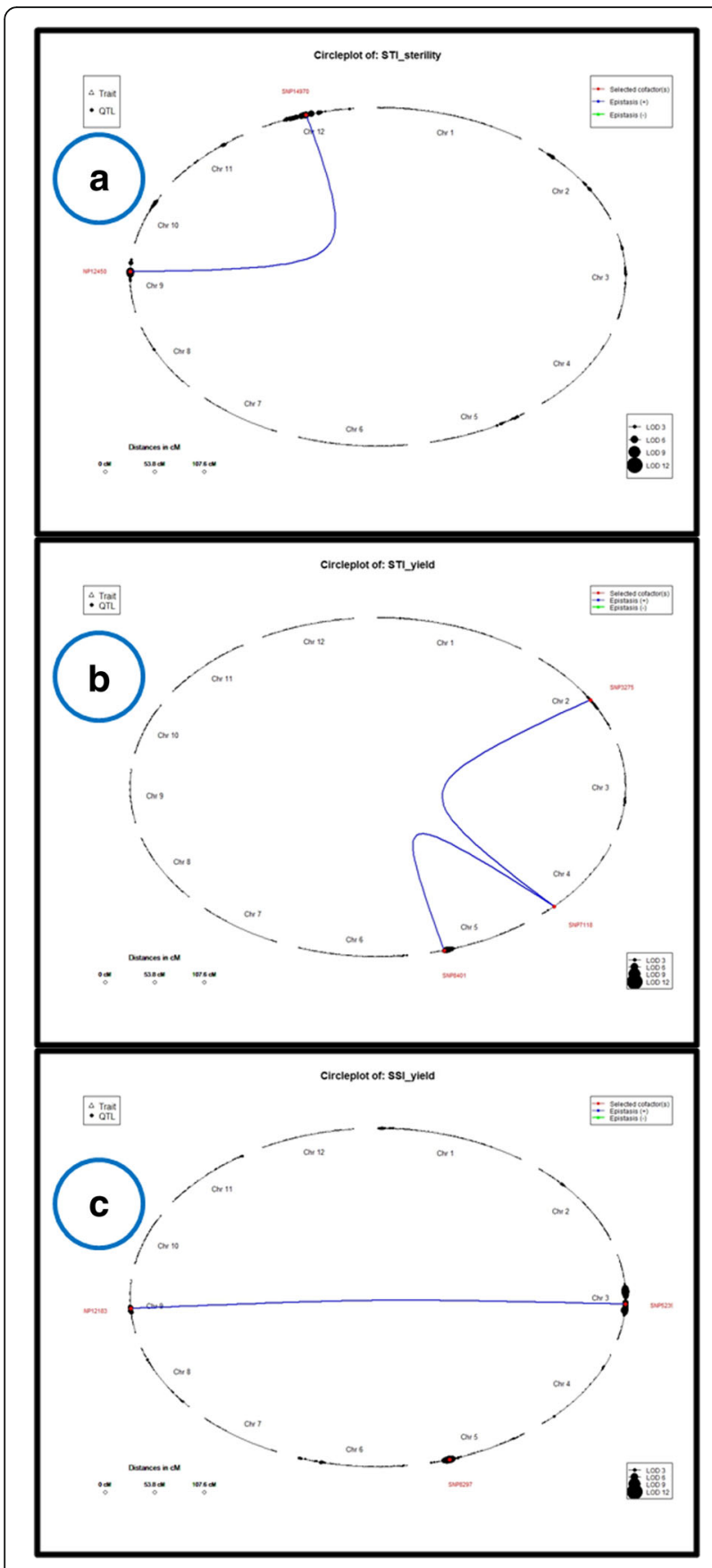

Fig. 5 Epistatic interaction network between SNP marker loci. a STI for sterility (b). STI for yield (c). SSI for yield

responsible for thermo-sensitive genic male sterility in HengnongS-1, one of the oldest and often-used TGMS line in indica two-line hybrid rice breeding programs in China (Qi et al. 2014). Also, Arabidopsis thaliana MALE STERILITY1 (MS1) gene encodes for a protein homologous to the PHD-finger class of transcription factor and has been demonstrated to be involved in tapetal development and pollen wall biosynthesis (Yang et al. 2007).
Glycosyltransferase attaches a single or multiple sugars to different bio-molecules and highly expresses in mature pollen grains and is involved in mature pollen grain formation in rice (Moon et al. 2013). GLYCOSYLTRANSFERASE1 (OsGT1) of rice present in qSTIPSS9.1 is involved in pollen wall formation, especially, the exine and intine construction and pollen maturation. The osgt1 mutant failed to produce mature pollen grains since its pollen had disrupted intine structure owing to low levels of starch and protein (Moon et al. 2013). Similarly, uneven pattern of exine 1 (upex1) gene of Arabidopsis encodes GT31 family glycosyltransferase in Arabidopsis and might be involved in galactosylation of arabinogalactan proteins (AGPs). The mutant of UPEX1 exhibit defective and irregular exine pattern and suggests that primexine localized AGPs could play a role in sporopollenin adhesion and patterning in early microspore wall development ( $\mathrm{Li}$ et al. 2016). HSFs are main players in imparting heat stress response by activating transcription of downstream genes including HSPs (Guo et al. 2008). AtHsfB4 has a role in root development in Arabidopsis and involved in early stage of heat shock (Lohmann et al. 2004; Begum et al. 2013). A similar OsHsf has been identified in this major QTL on chromosome 9 , which is yet to be characterized in rice.

Microtubule-associated proteins play a crucial role in the regulation of microtubule dynamics, and important for plant cell and organ development (Liu et al. 2013). The $65-\mathrm{kD}$ microtubule-associated protein (MAP65) family member in Arabidopsis (AtMAP65-1) is ubiquitously expressed during the cell cycle and in all plant organs and tissues with the exception of anthers and petals (Smertenko et al. 2004). However, Microtubuleassociated protein MAP65-1a (LOC_Os09g27700) of rice is expressed in anther and pistil. This might be indicative of its role in reproductive organ development in rice and hence is a good candidate for further studies in rice. Annexin functions to counteract oxidative stress, maintain cell redox homeostasis, and enhance drought tolerance (Szalonek 2015). Down-regulation of Arabidopsis annexin5 (Ann5) in transgenic Ann5-RNAi lines caused sterile pollen grains. Ann 5 is involved in pollen grain development, germination and pollen tube growth through the promotion of endo-membrane trafficking modulated by calcium (Zhu et al. 2014). TaAnn10 in wheat is highly expressed in floral bracts, pistil, anthers and immature endosperm and it correlates with anther development. But it fails to be induced by low temperature in thermosensitive genic male sterile lines, suggesting that specific down-regulation of TaAnn10 is associated with cold induced male sterility in wheat (Xu et al. 2016). The relative expression levels of TaAnn10 in the stamen strongly correlated with male fertility in recovery lines (Xu et al. 2016). One such 
Table 5 Epistatic interaction network

\begin{tabular}{|c|c|c|c|c|c|c|c|}
\hline S. No. & Traits & Interaction & SNPS & Chr. & Locus & Physical position (bp) & Annotation \\
\hline \multirow[t]{2}{*}{1} & STI sterility & SNP12453-SNP14970 & SNP12453 & 9 & LOC_Os09g29160.1 & $17,733,252-17,731,555$ & expressed protein \\
\hline & & & SNP14970 & 12 & LOC_Os12g24090.1 & $13,706,157-13,705,072$ & expressed protein \\
\hline \multirow[t]{3}{*}{2} & STI yield & SNP8401-SNP7118 & SNP8401 & 5 & LOC_Os05g44320.1 & $25,793,638-25,788,753$ & $\begin{array}{l}\text { WD domain, G-beta repeat domain } \\
\text { containing protein, expressed }\end{array}$ \\
\hline & & & SNP7118 & 4 & LOC_Os04g52280.1 & $31,069,545-31,075,601$ & dehydrogenase, putative, expressed \\
\hline & & SNP7118-SNP3275 & SNP3275 & 2 & LOC_Os02g37380.1 & $22,580,613-22,579,160$ & expressed protein \\
\hline \multirow[t]{2}{*}{3} & SSI yield & SNP5239-SNP12183 & SNP5239 & 3 & LOC_Os03g38450.1 & $21,344,825-21,346,870$ & expressed protein \\
\hline & & & SNP12183 & 9 & LOC_Os09g18230.1 & $11,184,420-11,171,639$ & expressed protein \\
\hline
\end{tabular}

annexin 10 (OsANN10/ LOC_Os09g27990) is present in the QTL interval qSTIPSS9.1.

A common response of organisms to drought, salinity, and temperature stresses is the accumulation of sugars and compatible solutes including trehalose. The increased trehalose accumulation correlates with elevated capacity for photosynthesis under both stress and nonstress conditions in rice (Garg et al. 2002). Trehalose-6phosphate synthase (TPS) plays an important role in trehalose metabolism and signalling. Overexpression of the trehalose-6-phosphate synthase gene OsTPS1 enhances the tolerance of rice seedling to cold, high salinity and drought stress without other significant phenotypic changes (Li et al. 2011). Similarly, the overexpression of trehalose-6-phosphate phosphatase in maize ears increases both kernel set and harvest index in drought stress condition. Increase in yield to the tune of $9 \%$ to $49 \%$ under non-drought or mild-drought conditions, and $31 \%-123 \%$ under more severe drought conditions, relative to yields from non-transgenic controls was observed (Nuccio et al. 2015). Similarly, trehalose concentration increased upon $4 \mathrm{~h}$ of heat stress at $40{ }^{\circ} \mathrm{C}$ and 4 days after cold stress at $4{ }^{\circ} \mathrm{C}$ in Arabidopsis thaliana (Kaplan et al. 2004). Over-expression of ScTPS1 and ScTPS2 under stress associated $r d 29 A$ promoter provided protection against drought, salt, freezing, and heat stress (Miranda et al. 2007). Hence, trehalose synthase and trehalose phosphate synthase are a probable candidate genes underlying QTL qSSIY5.1/qSTIY5.1 for yield under heat stress.

Our analysis for non-synonymous SNPs between the parents in the candidate genes like PTC1, tms9-1/OsMS1, OsGT1,MAP65-1a (LOC_Os09g27700), OsANN10, trehalose synthase, trehalose phosphate synthase, OsCR4, pectin methyl esterase and tesmin could not find allelic variants. This could be due to low coverage of the sequence data in either or both of the parents or lack of variation present in coding regions in the above genes. Further, the InDel polymorphism for these genes is not known. Alternatively, there could be variations in promoter, intron-exon junctions and UTR regions, which are not yet known. Hence, future effort is required to deep sequence these regions in the parents to identify polymorphisms, if any, in candidate genes. Nevertheless, the listed SNPs between N22 and IR64 can be utilised for fine mapping and functional validation of the QTLs.

The interaction network analysis showed evidence for the involvement of the major QTL, qSSIY5.1/qSTIY5.1, in digenic interactions, strengthening the role of this region in imparting heat tolerance. Though most of the SNPs involved in epistasis were in genes encoding uncharacterized expressed proteins, two SNPs were from known proteins coding genes namely, WD domain, G-beta repeat domain containing gene (LOC_Os05g44320) and dehydrogenase gene (LOC_Os04g52280). The WD40 protein is reported to play a role in diverse protein-protein interactions or protein-DNA interactions by acting as scaffolding molecule and promoting protein activity and thus functioning as a positive regulator of plant responses to various abiotic stresses such as salinity, osmotic and dehydration stress in plants (Mishra et al. 2012; Kong et al. 2015). Further, it is involved in various biological process, viz., signal transduction, gene transcriptional regulation, protein modifications, cytoskeleton assembly, vesicular trafficking, DNA damage and repair, cell death and cell cycle progression (Zhang and Zhang 2015).

Table 6 Non-synonymous SNPs between N22 and IR64 in the mapped QTL region for heat stress tolerance

\begin{tabular}{|c|c|c|c|c|c|}
\hline \multirow[t]{2}{*}{ S. No. } & \multirow{2}{*}{ QTLS } & \multirow[t]{2}{*}{ No. of genes with SNPS } & \multirow[t]{2}{*}{ No. of SNPS } & \multicolumn{2}{|c|}{ Non-synonymous SNPs between N22 and IR6 } \\
\hline & & & & Transition & Transversion \\
\hline 1 & qstipss9.1 & 16 & 28 & 23 & 5 \\
\hline 2 & qssipss 12.1 & 26 & 71 & 58 & 13 \\
\hline 3 & qstiy5.1 \& qssiy5.2 & 14 & 21 & 16 & 5 \\
\hline 4 & qssiy3.1 & 26 & 46 & 30 & 16 \\
\hline
\end{tabular}




\section{Conclusions}

Present study using Nagina22/IR64 RIL mapping population and a 5K SNP genotyping chip, identified a major novel QTL qSTIPSS9.1 for reproductive stage heat tolerance in a $394 \mathrm{kbp}$ region of rice chromosome 9 . The study also confirmed the presence of a known major QTL for heat tolerance on chromosome 5 (qSTIY5.1/ qSSIY5.1), which was narrowed down from $23 \mathrm{Mb}$ in the original study to a much smaller interval of 331 $\mathrm{kbp}$. This QTL was also involved in digenic interaction. Though the polymorphism survey in the candidate genes using the available data did not produce any trait linked variation, the SNPs identified could be useful in fine mapping. Further sequencing and functional validation is required for the identification of actual genes in these QTL regions responsible for the heat tolerance. Nonetheless, the two major QTLs identified here can be employed directly for crop improvement by marker assisted selection (MAS) after development of suitable scorable markers for breeding of high yielding heat tolerant rice varieties.

\section{Additional files}

Additional file 1: Figure. S1. Atmospheric temperature at the experimental location during heat stress treatment. (TIFF $584 \mathrm{~kb}$ )

Additional file 2: Table S1. List of genes in the five QTL intervals identified. Table S2. Non-synonymous SNPs in the mapped QTL intervals. Table S3. Reported QTLs related to heat stress tolerance in rice. (XLSX $52 \mathrm{~kb}$ )

\section{Acknowledgements}

The first author is grateful to the Department of Science and Technology, New Delhi, India for providing financial assistance in the form of INSPIREfellowship. The research work was funded by National Agricultural Innovation Program (NAIP) under the project "Bioprospecting of genes and allele mining for abiotic stress tolerance".

\section{Authors' contributions}

The research material developed by TM was advanced and maintained by PSS, CP and SVAM. Phenotyping was carried out by PSS and MKR. Genotyping was carried out by SVAM. Marker analysis was carried out by PSS and CP. QTL mapping was carried out by PSS and SVAM. In-silico search for putative genes in QTL intervals was carried out by PSS. Epistatic interaction was found by CP. PSS compiled and interpreted the results. RT provided and maintained the phenotypic facility. PSS and SVAM drafted the manuscript. NKS and TM supervised the work. NKS edited and approved the manuscript. All the authors read and approved the manuscript.

\section{Competing interests}

The authors declare that they have no competing interest.

\section{Publisher's Note}

Springer Nature remains neutral with regard to jurisdictional claims in published maps and institutional affiliations.

\section{Author details}

${ }^{1}$ ICAR-National Research Centre on Plant Biotechnology, New Delhi, India ${ }^{2}$ Present address, Division of Plant Biotechnology, ICAR-Indian Institute of Pulses Research, Kanpur 208 024, India. ${ }^{3}$ ICAR-Indian Institute of Wheat and Barley Research, Karnal 132 001, India. ${ }^{4}$ Indian Council of Agricultural Research, Krishi Bhavan, New Delhi 110 001, India.
Received: 29 October 2016 Accepted: 30 May 2017

Published online: 05 June 2017

\section{References}

Begum T, Reuter R, Schöffl F (2013) Overexpression of AtHsfB4 induces specific effects on root development of Arabidopsis. Mech Dev 130(1):54-60. doi:10. 1016/j.mod.2012.05.008

Broman KW, Wu H, Sen Ś, Churchill GA (2003) R/qtl: QTL mapping in experimental crosses. Bioinformatics 19(7):889-890

Buu BC, Ha PTT, Tam BP, Nhien TT, Hieu NV, Phuoc NT, Minh LT, Giang LH, Lang NT (2014) Quantitative trait loci associated with heat tolerance in rice (Oryza sativa L.) Plant Breed Biotech 2:14-24

Cao L, Zhao J, Zhan X, Li D, He L, Cheng S (2003) Mapping QTLs for heat tolerance and correlation between heat tolerance and photosynthetic rate in rice. Chin J Rice Sci 17:223-227

Carriger S, Vallee D (2007) More crop per drop. Rice Today 6:10-13

Chang-Lan Z, Ying-Hui X, Chun-Ming W, Ling J, Hu-Qu Z, Jian-Min W (2005) Mapping QTL for heat-tolerance at grain filling stage in rice. Rice Sci 12(1):33-38

Chen M, Presting G, Barbazuk WB, Goicoechea JL, Blackmon B, Fang G, Kim H, Frisch D, Yu Y, Sun S, Higingbottom S, Phimphilai J, Phimphilai D, Thurmond S, Gaudette B, Li P, Liu J, Hatfield J, Main D, Farrar K, Henderson C, Barnett L, Costa R, Williams B, Walser S, Atkins M, Hall C, Budiman MA, Tomkins JP, Luo M, Bancroft I, Salse J, Regad F, Mohapatra T, Singh NK, Tyagi AK, Soderlund C, Dean RA, Wing RA (2002) An integrated physical and genetic map of the rice genome. Plant Cell 14(3):537-545

Chen Q, Yu S, Li C, Mou T (2008) Identification of QTLs for heat tolerance at flowering stage in rice. Sci Agric Sin 41:315-321

Cheng L, Wang J, Uzokwe V, Meng L, Wang Y, Sun Y, Zhu L, Xu J, Li Z (2012) Genetic analysis of cold tolerance at seedling stage and heat tolerance at anthesis in rice (Oryza sativa L.) J Integ Agriculture 11:359-367

Doyle JJ, Doyle JL (1990) Isolation of plant DNA from fresh tissue. Focus 12:13-15

Fernandez GCJ (1992) Effective selection criteria for assessing plant stress tolerance. In: Kus EG (ed) Adaptation of Food Crop Temperature and Water Stress. Proceeding of 4th International Symposium, Asian Vegetable and Research and Development Center, Shantana, Taiwan, pp 257-270

Fischer RA, Maurer R (1978) Drought resistance in spring wheat cultivars.I. Grain yield responses. Aust J Agric Res 29:892-912

Garg AK, Kim JK, Owens TG, Ranwala AP, Choi YD, Kochian LV, Wu RJ (2002) Trehalose accumulation in rice plants confers high tolerance levels to different abiotic stresses. PNAS 99(10):15898-15903. doi:10.1073/pnas.252637799

Giorno F, Wolters-Arts M, Mariani C, Ivo R (2013) Ensuring reproduction at high temperatures: the heat stress response during anther and pollen development. Plants 2:489-506. doi:10.3390/plants2030489

Guo J, Wu J, Ji Q, Wang C, Luo L, Yuan Y, Wang Y, Wang J (2008) Genome-wide analysis of heat shock transcription factor families in rice and Arabidopsis. Genet Genomics 35(2):105-118. doi:10.1016/S1673-8527(08)60016-8

IPCC (2007) Summary for Policymakers. In: Solomon S, Qin D, Manning M, Chen Z, Marquis M, Averyt KB, Tignor M, Miller HL (eds) Climate change 2007: The Physical Science Basis. Contribution of working group I to the fourth assessment report of the intergovernmental panel on climate change. Cambridge University Press, Cambridge, pp 1-18

Jagadish SVK, Cairns J, Lafitte R, Wheeler TR, Price AH, Craufurd PQ (2010b) Genetic analysis of heat tolerance at anthesis in rice. Crop Sci 50:1633-1641

Jagadish SVK, Craufurd PQ, Wheeler TR (2007) High temperature stress and spikelet fertility in rice (Oryza sativa L.) J Exp Bot 58(7):1627-1635

Jagadish SVK, Muthurajan R, Oane R, Wheeler TR, Heuer S, Bennett J, Craufurd PQ (2010a) Physiological and proteomic approaches to address heat tolerance during anthesis in rice (Oryza sativa L.) J Exp Bot 61:143-156. doi:10.1093/jxb/erp289

Jagadish SVK, Craufurd PQ, Wheeler TR (2008) Phenotyping parents of mapping populations of rice (Oryza sativa L.) for heat tolerance during anthesis. Crop Sci 48:1140-1146

Kaplan F, Kopka J, Haskell DW, Zhao W, Schiller KC, Gatzke N, Sung DY, Guy $\mathrm{CL}$ (2004) Exploring the temperature-stress metabolome of Arabidopsis. Plant Physiol 136(4):4159-4168

Kong D, Li M, Dong Z, Ji H, Li X (2015) Identification of TaWD40D, a wheat WD40 repeat-containing protein that is associated with plant tolerance to abiotic stresses. Plant Cell Rep 3:395-410

Kumar V, Singh A, Mithra SVA, Krishnamurthy SL, Parida SK, Jain S, Tiwari KK, Kumar P, Rao AR, Sharma SK, Khurana JP, Singh NK, Mohapatra T (2015) 
Genome-wide association mapping of salinity tolerance in rice (Oryza sativa L). DNA Res 22(2):133-145. doi:10.1093/dnares/dsu046

Li H, Yuan Z, Vizcay-Barrena G, Yang C, Liang W, Zong J, Wilson ZA, Zhang D (2011a) PERSISTENT TAPETAL CELL1 encodes a PHD-finger protein that is required for tapetal cell death and pollen development in rice. Plant Physiol 156(2):615-630. doi:10.1104/pp.111.175760

Li HW, Zang BS, Deng XW, Wang XP (2011b) Overexpression of the trehalose-6phosphate synthase gene OsTPS1 enhances abiotic stress tolerance in rice. Planta 234(5):1007-1018. doi:10.1007/s00425-011-1458-0

Li WL, Liu Y, Douglas CJ (2016) Role of glycosyltransferases in pollen wall primexine formation and exine patterning. Plant Physiol. 173;(1):167-82.

Li N, Zhang DS, Liu HS, Yin CS, Li XX, Liang WQ, Yuan Z, Xu B, Chu HW, Wang J, Wen TQ, Huang H, Luo D, Ma H, Zhang DB (2006) The rice tapetum degeneration retardation gene is required for tapetum degradation and anther development. Plant Cell 18:2999-3014

Liu C, Qi X, Zhao Q, Yu J (2013) Characterization and functional analysis of the potato pollen-specific Microtubule-Associated Protein SBgLR in Tobacco. PLoS One 8(3):e60543. doi:10.1371/journal.pone.0060543

Liu JX, Liao DQ, Oane R, Estenor L, Yang XE, Li ZC, Bennett J (2006) Genetic variation in the sensitivity of anther dehiscence to drought stress in rice. Field Crop Res 97:87-100

Lobell DB, Schlenker W, Costa-Roberts J (2011) Climate trends and global crop production since 1980. Science 333:616-620

Lohmann C, Eggers-Schumacher G, Wunderlich M, Schöffl F (2004) Two different heat shock transcription factors regulate immediate early expression of stress genes in Arabidopsis. Mol Gen Genomics 271(1):11-21

Mansueto L, Fuentes RR, Borja FN, Detras J, Abriol-santos M, ChebotarovD, ... Alexandrov N (2017) Rice SNP-seek database update: new SNPs, InDels, and queries. Nucleic Acids Res 45:D1075-D1081.

Matsui T, Omasa K, Horie T (1997) High temperature induced spikelet sterility of japonica rice at flowering in relation to air humidity and wind velocity conditions. Japan Journal of Crop Science 66:449-455

Matsui T, Omasa K, Horie T (1999) Rapid swelling of pollen grains in response to floret opening unfolds locule in rice. Plant Production Science 2(3):196-199

Matsui T, Omasa K (2002) Rice (Oryza sativa L.) cultivars tolerant to high temperature at flowering: anther characteristics. Ann Bot 89(6):683-637

Meng L, Li H, Zhang L, Wang J (2015) QTL IciMapping: integrated software for genetic linkage map construction and quantitative trait locus mapping in bi-parental populations. Crop J 3:265-279. doi:10.1016/j.cj.2015.01.001

Miranda JA, Avonce N, Suárez R, Thevelein JM, Van Dijck P, Iturriaga G (2007) A bifunctional TPS-TPP enzyme from yeast confers tolerance to multiple and extreme abiotic-stress conditions in transgenic Arabidopsis. Planta 226(6):1411-1421

Mishra AK, Puranik S, Prasad M (2012) Structure and regulatory networks of WD40 protein in plants. J Plant Biochem Biotechnol 21:32-39

Mohapatra T, Robin S, Sarla N, Sheshashayee M, Singh AK, Singh K, Singh NK, Amitha Mithra SV, Sharma RP (2014) EMS induced mutants of upland rice variety Nagina22: generation and characterization. Proc Indian National Science Academy 80:163-172

Moon S, Kim SR, Zhao G, Yi J, Yoo Y, Jin P, Lee SW, Jung KH, Zhang D, An G (2013) Rice GLYCOSYLTRANSFERASE1 encodes a glycosyltransferase essential for pollen wall formation. Plant Physiol 161(2):663-675. doi:10.1104/pp.112.210948

Nakagawa H, Horie T, Matsui T (2002) Effects of climate change on rice production and adaptive technologies. In: Mew TW, Brar DS, Peng S, Dawe D, Hardy B (eds) Rice science: innovations and impact for livelihood China: International Rice Research Institute, pp 635-657

Nuccio ML, Wu J, Mowers R, Zhou HP, Meghji M, Primavesi LF, Paul MJ, Chen X Gao Y, Haque E, Basu SS, Lagrimini LM (2015) Expression of trehalose-6phosphate phosphatase in maize ears improves yield in well-watered and drought conditions. Nat Biotech 33:862-869. doi:10.1038/nbt.3277

Pandit A, Rai V, Bal S, Sinha S, Kumar V, Chauhan M, Gautam RK, Singh R, Sharma PC, Singh AK, Gaikwad K, Sharma TR, Mohapatra T, Singh NK (2010) Combining QTL mapping and transcriptome profiling of bulked RILs for identification of functional polymorphism for salt tolerance genes in rice (Oryza sativa L.) Mol Gen Genomics 284(2):121-136. doi:10.1007/s00438-010-0551-6

Poli Y, Basava RK, Panigrahy M, Vinukonda VP, Dokula NR, Voleti SR, Desiraju S, Neelamraju S (2013) Characterization of a Nagina22 rice mutant for heat tolerance and mapping for yield traits. Rice 6:36

Prakash C, Mithra SVA, Singh PK, Mohapatra T, Singh NK (2016) Unravelling the molecular basis of oxidative stress management in a drought tolerant rice genotype Nagina22. BMC Genomics 17:774. doi:10.1186/s12864-016-3131-2
Prasad PW, Boote KJ, Jr LHA, Sheehy JE, Thomas JMG (2006) Species, ecotype and cultivar differences in spikelet fertility and harvest index of rice in response to high temperature stress. Field Crop Res 95:398-411

Qi Y, Liu Q, Zhang L, Mao B, Yan D, Jin Q, He Z (2014) Fine mapping and candidate gene analysis of the novel thermo-sensitive genic male sterility tms9-1 gene in rice. Theor Appl Genet 127(5):1173-1182. doi:10.1007/s00122-014-2289-8

Raman A, Verulkar SB, Mandal N, Variar M, Shukla VD, Dwivedi JL, Singh BN Singh ON, Swain P, Mall AK, Robin S, Chandrababu R, Jain A, Ram T, Hittalmani S, Haefele S, Piepho HP, Kumar A (2012) Drought yield index to select high yielding rice lines under different drought stress severities. Rice 5 : 31. doi:10.1186/1939-8433-5-31

Sarwar M, Avesi GM (1985) Evaluation of rice germplasm for high temperature tolerance. Pakistan J Agric Res 6(3):162-164

Sato K, Inaba K, Tosawa M (1973) High temperature injury of ripening in rice plant. The effects of high temperature treatment at different stages of panicle development on the ripening. Proceedings of Crop Science Society of Japan 42:207-213

Smertenko AP, Chang HY, Wagner V, Kaloriti D, Fenyk S, Sonobe S, Lloyd C, Hauser MT, Hussey PJ (2004) The Arabidopsis Microtubule-Associated Protein AtMAP65-1: molecular analysis of its microtubule bundling activity. Plant Cell 16(8):2035-2047

Szalonek M, Sierpien B, Rymaszewski W, Gieczewska K, Garstka M, Lichocka M, Sass L, Paul K, Vass I, Vankova R, Dobrev P, SzczesnyP MW, Krusiewicz D, Strzelczyk-Zyta D, Hennig J, Konopka-Postupolska D (2015) Potato Annexin STANN1 promotes drought tolerance and mitigates light stress in transgenic Solanum tuberosum L. Plants. PLoS One 10(7):e0132683. doi:10.1371/journal. pone.0132683

Tazib T, Kobayashi Y, Koyama H, Matsui T (2015) QTL analyses for anther length and dehiscence at flowering as traits for the tolerance of extreme temperatures in rice (Oryza sativa L.) Euphytica 203:629-642

Tiwari S, Krishnamurthy SL, Kumar V, Singh B, Rao A, Mithra SVA, Rai V, Singh AK, Singh NK (2016) Mapping QTLs for salt tolerance in rice (Oryza sativa L.) by Bulked Segregant Analysis of Recombinant Inbred Lines using 50K SNP Chip. PLoS One 11(4):e0153610. doi:10.1371/journal.pone.0153610

United Nations, Department of Economic and Social Affairs, Population Division (2011) World Population Prospects: The 2010 Revision, Volume I: Comprehensive Tables. ST/ESA/SER.A/313

Xiao Y, Pan Y, Luo L, Zhang G, Deng H, Dai L, Liu X, Tang W, Chen L, Wang GL (2011a) Quantitative trait loci associated with seed set under high temperature stress at the flowering stage in rice (Oryza sativa L.) Euphytica 178:331-338. doi:10.1007/s10681-010-0300-2

Xiao YH, Pan Y, Luo LH, Deng HB, Zhang GL, Tang WB, Chen LY (2011b) Quantitative trait loci associated with pollen fertility under high temperature stress at flowering stage in rice (Oryza sativa L). Rice Sci 18(2):1-7

Xu L, Tang Y, Gao S, Su S, Hong L, Wang W, Fang Z, Li X, Ma J, Quan W, Sun H, Li X, Wang Y, Liao X, Gao J, Zhang F, Li L, Zhao C (2016) Comprehensive analyses of the annexin gene family in wheat. BMC Genomics 17:415. doi:10.1186/s12864-016-2750-y

Yang C, Vizcay-Barrena C, Conner K, Wilson ZA (2007) MALE STERILITY1 is required for tapetal development and pollen wall biosynthesis. Plant Cell 19(11):3530-3548

Ye C, Argayoso MA, Redoña ED, Sierra SL, Laza MA, Dilla CJ, Mo Y, Thomson MJ, Chin J, Delaviña CB, Diaz GQ, Hernandez JE (2012) Mapping QTL for heat tolerance at flowering stage in rice using SNP markers. Plant Breed 131(1):33-41

Ye C, Tenorio FA, Argayoso MA, Laza MA, Koh HJ, Redoña ED, Jagadish KS, Gregorio GB (2015) Identifying and confirming quantitative trait loci associated with heat tolerance at flowering stage in different rice populations. BMC Genet 16:41

Zhang C, Zhang F (2015) The Multifunctions of WD40 Proteins in genome integrity and cell cycle progression. J Genomics 3:40-50

Zhang G, Chen L, Xiao G, Xiao Y, Chen X, Zhang S (2009) Bulked segregant analysis to detect QTL related to heat tolerance in rice using SSR markers. Agric Sci China 8:482-487

Zhang T, Yang L, Jiang K, Huang M, Sun Q, Chen W, Zheng J (2008) QTL mapping for heat tolerance of the tassel period of rice. Mol Plant Breed 6:867-873

Zhao L, Lei J, Huang Y, Zhu S, Chen H, Huang R, Peng Z, Tu Q, Shen X, Yan S (2016) Mapping quantitative trait loci for heat tolerance at anthesis in rice using chromosomal segment substitution lines. Breeding Science Preview. doi:10.1270/jsbbs.15084.

Zhu J, Wu X, Yuan S, Qian D, Nan Q, An L, Xiang Y (2014) Annexin5 Plays a Vital Role in Arabidopsis pollen development via $\mathrm{Ca}^{2+}$-dependent membrane trafficking. PLoS One 9(7):e102407 